was 70 days. Of all the re-admission, 99 were breastfeeding on re-admission, 24 were mixed feeds and 19 bottle fed.

Conclusions The two main reasons for readmissions were jaundice and weight loss. There was an overlap of this because a proportion of jaundiced babies were noted to have weight loss or poor feeding on readmission. We believe that these are potentially avoidable readmissions if there was sufficient feeding support for the mother and babies in the community. It would alleviate the additional emotional stress imposed on the mother and family by the readmission. It would also reduce the impact on the bed occupancy on an already stretched maternity and transitional care wards.

\section{GP270 HEALTH PROBLEMS IN NEONATES WITH PERINATAL HIV EXPOSURE}

${ }^{1}$ Svetlana Shugaeva, ${ }^{2}$ Alla Petrova, ${ }^{2}$ Anastasiya Vanyarkina*, 2 Lyubov Rychkova. ${ }^{2}$ rrkutsk State Medical University, Irkutsk, Russian Federation; ${ }^{2}$ Scientific Centre for Family Health and Human Reproduction Problems, Irkutsk, Russian Federation

10.1136/archdischild-2019-epa.329

Introduction Antenatal and perinatal periods determine the potential of a child's health for near and distant future. Considering the increasing number of HIV-exposed uninfected newborns (especially in areas of high risk of HIV infection), the aim of our study was to determine the prevalence of health problems of newborns who avoided perinatal HIV transmission.

Methods We conducted a cohort randomized study that included 177 children from birth to 18 months of life. The sample was divided in two groups, equivalent in sex: the main group ( $\mathrm{n}=87$, random sample) comprised neonates with perinatal HIV exposure, the comparison group included children with no HIV exposure (matched sample). Exclusion criteria were confirmed HIV infection $(\mathrm{n}=3)$, and parents' refusal to participate $(\mathrm{n}=0)$. We evaluated the frequency $\left(\mathrm{P}_{\mathrm{abs}} / \mathrm{P} \%\right)$ of seven signs (prematurity, intrauterine growth retardation, neonatal abstinence syndrome, abnormalities of postnatal adaptation, perinatal damage of CNS, congenital and perinatally-acquired infections, congenital malformations and/or minor developmental abnormality). A comparative analysis was performed using the $\chi 2$ criterion and its modifications (Yates corrected Chi-square, two-tailed Fisher exact p), relative risk index was calculated (RR [CI0.95]).

Results Health problems in neonates according to the analyzed signs were significantly more frequent in main group (76/ $87.6 \%)$ than in comparison group $(28 / 31.1 \%) \quad(\mathrm{RR}=2.8$ $[2.0-3.9] ; \chi 2=57.75, \mathrm{p}<0.0001)$.

In contrast with children, not exposed to HIV, children of HIV-infected mothers showed statistically significant incidence of the following signs: intrauterine growth retardation: 26/ $29.9 \%$ vs $5 / 5.5 \% \quad(\mathrm{RR}=5.4[2.2-13.4] ; \chi 2=16.5, \mathrm{p}$ $<0.0001)$; abnormalities of postnatal adaptation: $14 / 16.1 \%$ vs $3 / 3.3 \%(\mathrm{RR}=4.8[1.4-16.2] ;-, \mathrm{p}=0.005)$; congenital and perinatally-acquired infections: $17 / 19.5 \%$ vs $7 / 7.8 \% \quad(\mathrm{RR}=$ $2.5[1.1-5.7] ; \chi 2=5.2, \mathrm{p}=0.022)$; neonatal abstinence syndrome $12 / 13.8 \%$ vs $0 / 0 \% \quad(\mathrm{RR}=25.9$ [1.6-430.1] - calculated by L.A. Pevnitsky's method; -, p <0.0001); perinatal CNS damage: $30 / 34.5 \%$ vs $15 / 16.7 \% \quad(\mathrm{RR}=2.1 \quad[1.2-3.6]$; $\left.\chi^{2}=7.4, \mathrm{p}=0.007\right)$.

The most severe problems (frequency of occurrence and the number of registered conditions) were found in HIV- exposed children in families with low income and families where adults had alcohol and/or drug dependence.

Conclusions Health problems, relating to disorders in intrauterine growth and early postnatal period are registered mostly in HIV-exposed uninfected children, born to HIV-positive mothers. Increased incidence of health problems and their concentration in low-income families with deviant lifestyle requires careful approach by pediatricians.

\section{GP271 AN AUDIT OF A TERTIARY NEONATAL CENTRE'S TRANSITION FROM INDIVIDUALISED TO STOCK PARENTERAL NUTRITION IN EXTREMELY PRETERM INFANTS}

James Trayer* , Anne Twomey. National Maternity Hospital, Dublin, Ireland

10.1136/archdischild-2019-epa.330

Introduction Infants $\leq 28$ weeks gestational typically require parenteral nutrition (PN) while enteral feeds are slowly advanced. The National Maternity Hospital has recently commenced prescribing standardised $\mathrm{PN}$ for these infants (using new and more concentrated stock bags that are produced by Baxter Healthcare Ltd) rather than individualised PN (IPN). Our aim was to assess the clinical impact of this change.

Methods A retrospective chart review was conducted including all infants $\leq 28$ weeks gestation during a six month period from August 2018 to January 2019. The outcomes reviewed included subsequent need for IPN, electrolyte abnormalities, need for additional intravenous fluids to correct biochemical abnormalities, proportion of infants receiving the recommended nutritional requirements.

Results A total of 21 babies were included in the study. 7/21 (33\%) required a switch from SPN to IPN. The most common reason was hyponatraemia (3/7) followed by hypoglycaemia (2/7). Electrolyte abnormalities were seen in $13 / 21$ (62\%). The most common reason was hyponatraemia $(8 / 21)$ followed by hypernatraemia (4/21). Electrolyte abnormalities were also more commonly seen in infants <25wks $(7 / 8$, $88 \%)$. Supplemental fluids were required in 6/21 (29\%) babies - 3 for hyponatraemia and 2 for hypernatraemia. Daily targeted volumes of SPN to optimise nutrition were frequently missed, especially on D1, when only $2 / 21(10 \%)$ infants received the recommended volumes. This was largely due to a hospital based practice of using non-nutritive fluids to maintain the patency of the second lumen of an umbilical venous line $(12 / 21,57 \%)$. Other reasons included the need for fluid restriction $(5 / 21,24 \%)$ or the need for inotropes $(2 / 21,10 \%)$.

Conclusions and discussion The switch to SPN has reduced the need for IPN from $100 \%$ to $33 \%$. This represents a significant reduction in time spent prescribing IPN, a complex product associated with a high risk of prescription errors. The reduced reliance on IPN also represents a financial saving for the hospital. This audit highlighted the significant impact that a hospital based practice used to maintain patency of umbilical lines has on our ability to give optimal nutrition (both SPN and IPN) during the first few days of life. This practice now needs to be re-assessed in the light of this finding. Due attention also needs to be given sodium requirements when using these new SPN bags to avoid the risk of hypo/hypernatraemia. 